\title{
Baseline circulating stem-like cells predict survival in patients with metastatic breast Cancer
}

\author{
Chun-Hui Lee ${ }^{1 \dagger}$, Jason Chia-Hsun Hsieh ${ }^{2,3,4}$, Tyler Min-Hsien Wu ${ }^{2,4,5,6}$, Ting-Shiuan Yeh ${ }^{2}$, Hung-Ming Wang ${ }^{2,3}$, \\ Yung-Chan Lin 2,3, Jen-Shi Chen 2,3, Chia-Lin Lee ${ }^{7,8,9}$, Wen-Kuan Huang 2,3,10, Tsung-Min Hung ${ }^{3,11}$, Tzu-Tsen Yen ${ }^{3,12}$, \\ Sheng-Chieh Chan ${ }^{3,12}$, Wen-Chi Chou ${ }^{2,3}$, Feng-Che Kuan ${ }^{13}$, Ching-Chih Hu ${ }^{3,14}$ and Pei-Hung Chang ${ }^{2,3,15^{*}}$ (D)
}

\begin{abstract}
Background: Circulating tumor cells (CTCS) are associated with breast cancer prognosis. Research is limited regarding the role of circulating cancer stem-like cells (CCSCs) considering the treatment response and survival among patients with metastatic breast cancer. Accordingly, we performed this prospective study to clarify the prognostic significance of baseline cCSCs for metastatic breast cancer in terms of first-line chemotherapy.
\end{abstract}

Methods: Between April 2014 and January 2016, we prospectively enrolled 48 patients with stage IV breast invasive ductal carcinoma who underwent first-line chemotherapy. We identified and analyzed CTCs and CCSCs by using a protocol based on negative selection and flow cytometry before chemotherapy. CTCs were identified as EpCAM ${ }^{+}$Hoechst ${ }^{+}$CD $45^{-}$cells and cCSCs as CD $133^{+}$EpCAM $^{+}$Hoechst ${ }^{+}$CD $45^{-}$cells. cCSCs were expressed as a percentage of CTCS. The associations between CTCS, CCSCs, and the clinicopathological variables that were predictive of the treatment response and survival outcome were analyzed using univariate and multivariate analyses.

Results: We identified CTCs in all the enrolled patients, with a median number of 33.9/mL CTCs. CSCs were isolated in 97.9\% of the patients; the median percentage of CCSCs was 14.7\%. A high baseline level of CCSCs was correlated with an inferior tumor response rate (54.2\% vs. 95.8\%, $p<0.001)$, overall survival (OS; median: 27.7 months vs. not reached, $p<0.001$ ), and progression-free survival (PFS; median: 5.7 vs. 18.0 months, $p<0.001$ ). Multivariate analysis revealed that along with other clinical variables, baseline cCSCs remained an independent prognostic factor for OS and PFS.

Conclusions: Baseline cCSCs predict the treatment response as well as survival in patients with metastatic breast cancer undergoing first-line chemotherapy. Therefore, the measurement of CCSCs may assist in identifying early cancer treatment response and prognosis.

Keywords: Breast cancer, Chemotherapy, Circulating tumor cells, Cancer stem cells, CD133

\section{Background}

Breast cancer is a constituent of the most prevalent disease women may face [1]. Overall mortality for this disease decreased by approximately 40\% from 1989 to 2015 [1]. This decrease is possibly because of the result of increased awareness, early diagnosis through screening, and major

\footnotetext{
* Correspondence: ph555chang@gmail.com

${ }^{\dagger}$ Chun-Hui Lee and Jason Chia-Hsun Hsieh contributed equally to this work. ${ }^{2}$ Circulating Tumour Cell Lab, Division of Medical Oncology, Department of Internal Medicine, Chang Gung Memorial Hospital at Linkou, Taoyuan, Taiwan

${ }^{3}$ College of Medicine, Chang Gung University, Taoyuan, Taiwan

Full list of author information is available at the end of the article
}

treatment advances [1]. The long-term outcomes of metastatic breast cancer may be influenced by various biological features such as the age at initial diagnosis; status of hormone receptors (HRs), including estrogen receptor (ER), and/or progesterone receptor (PR), as well as human epidermal growth factor receptor 2 (HER-2); and the numbers and sites of metastases [2,3]. Chemotherapy, endocrine therapy, and targeted therapy remains the present standard of care is HER2-directed treatment combined with chemotherapy [4]. However, approximately $20-50 \%$ of patients do not exhibit a response to first-line treatment [5]. Moreover, the standard test for metastatic

(c) The Author(s). 2019 Open Access This article is distributed under the terms of the Creative Commons Attribution 4.0 International License (http://creativecommons.org/licenses/by/4.0/), which permits unrestricted use, distribution, and 
breast cancer, which involves functional and morphological imaging, does not provide sufficient prognostic information [6]. Therefore, the development of prognostic biomarkers is urgently required for patients undergoing chemotherapy to treat metastatic breast cancer.

Recently, liquid biopsy has shown promise for elucidating intratumoral and intertumoral heterogeneity. One of the most applicable liquid biopsies was established in 1869 , and it is used for identifying circulating tumor cells (CTCs) as well as circulating epithelial cells (CECs) [7]. CECs and CTCs are cells that are shed into the bloodstream from the primary tumor and express epithelial cell surface (CD) markers as well as tumorspecific markers, in addition to frequently expressing epithelial markers that include the epithelial cell adhesion molecule (EpCAM) or cytokeratins [8]. Nevertheless, CECs and CTCs do not typically express red and white blood cell markers, such as CD45 and CD235 [9, 10]. These cells are believed to have the ability to metastasize to remote organs, advance thrombosis, and gain resistance to anticancer drugs [11-13]. Studies have proven the prognostic significance of CTCs in patients with assorted types of solid tumors, including those with breast cancer [14-17]. However, a novel concept of cancer stem cells has emerged, resulting in new diagnostic procedures being developed [18]. CTCs that are collected from patients with metastatic breast cancer often exhibit the overexpression of stem cell markers, suggesting that metastasis is induced by a subpopulation of CTCs that express a cancer stem cell marker [19]. Numerous researchers have revealed that a particular subdivision of CTCs can express stem cell markers (e.g., CD133 [12] or CD44 [20]), can have cells with cancer stem cell characteristics [21, 22], and can thus be considered circulating cancer stem-like cells (cCSCs) [23]. Many studies have used different definitions of cCSCs, which we have defined as cells expressing both CD133 and EpCAM after CD45 depletion. CD133 is an indicator of cells that initiate tumors in numerous cancers [24, 25]; moreover, tumor cells that express CD133 demonstrate cancer stem cell properties, characterized by selfrenewal capabilities in culture, in order to differentiate into cells recapitulating initial breast cancer tumors and develop tumors in animal models [26]. Because the most commonly used marker for identifying cCSCs is CD133 [27], we included this marker to improve the ability to compare and validate our findings. cCSCs have been used to predict chemotherapy resistance in many cancers [28-30]. In a report, cCSCs were correlated with chemotherapy response and recurrence in nonmetastatic breast cancer [12]. Nevertheless, studies have yet to resolve the function of cCSCs in metastatic breast cancer, especially regarding whether these cells are associated with chemoresistance and survival. Therefore, we performed this prospective multicenter study, with the fundamental objective of evaluating the prognostic value of CTCs and CSCs in conjugation with clinical variables in patients diagnosed with metastatic breast cancer and undergoing first-line chemotherapy.

\section{Methods \\ Study design}

This prospective observational study examined the clinical importance of baseline cCSCs during first-line palliative chemotherapy for metastatic breast cancer. Our study endpoints were identifying the correlations between CTCs, CSCs, and clinicopathological variables with the treatment response rate, progression-free survival (PFS), and overall survival (OS). After determine the response to treatment, in survival analysis, we identified disease progression as well as death from any cause. In addition, we designed the analysis to be performed after more than half of the disease progression events had taken place. We report the derived study results in accordance with the guidelines outlined in the Reporting Recommendations for Tumor Marker Prognostic Studies (REMARK).

\section{Patient enrollment and Cancer status evaluation}

This study was conducted at two medical centers: the Linkou and Keelung branches of Chang Gung Memorial Hospital, Taiwan. Our applied study protocol was approved by the Institutional Review Board of Chang Gung Memorial Hospital (approval ID: 103-0425B and 103-5322B). For protocols that required ethical approval, written informed consent was obtained from all patients. Eligible patients had breast invasive ductal carcinoma, confirmed by histologic or cytopathologic findings, that was surgically unresectable and/or metastatic (stage IV, according to the 7th edition of the staging manual of the American Joint Committee on Cancer [AJCC]). Other enrollment were included the following: (1) patients aged $\geq 20$ years; (2) those with the ability to understand the contents of the consent form and sign it of their own accord; (3) those having satisfactory renal and liver function and blood cell counts to undergo chemotherapy; and (4) thoses who had experienced endocrine therapy failure in those with a positive HR status. We excluded patients with synchronous cancer and thoses who had cancer in the 5 years prior to enrollment. All patients were subjected to baseline evaluations, including evaluations of the clinical history, demographic data, computed tomography $(\mathrm{CT})$ scan, pathological characteristics, and biochemical evaluation. For executing disease staging and management procedures used in the study, we adhered to standard treatment protocols in accordance with institutional guidelines. Systemic anticancer therapy consisted of trastuzumab plus docetaxel, paclitaxel plus gemcitabine, and doxorubicin plus cyclophosphamide, contingent upon the decision made by the physician. We 
executed evaluations of tumor response by using CT and/ or positron emission tomography on the basis of the Response Evaluation Criteria in Solid Tumors (RECIST) version 1.1 guidelines [31]; the multidisciplinary breast tumor board ultimately decided the tumor response. PFS and OS were calculated from the date on which the first cycle of palliative chemotherapy was administered to that of disease progression or death following chemotherapy. We followed the entirety of patients until death or September 30, 2017.

\section{Identifying CTCs and CCSCs}

We identified CTCs by using positive detection and negative selection strategies, and we have validated them in our previously executed studies [32, 33]. The methods adopted included the following: (1) a negative selection protocol that entails the use of a CD45 depletion kit to deplete red blood cells using lysis and leukocytes; and (2) a process of flow cytometry for quantitatively identifying CTCs $\left(\mathrm{EpCAM}^{+}\right.$Hoechst $\left.^{+} \mathrm{CD} 45^{-}\right)$and cCSCs $\left(\mathrm{CD} 133^{+}\right.$ $\mathrm{EpCAM}^{+} \mathrm{Hoechst}^{+} \mathrm{CD} 45^{-}$), and then calculating their numbers. cCSCs were expressed as a percentage of CTCs. CTC tests were performed using peripheral blood $(4 \mathrm{~mL})$ after disposing of the initial $4 \mathrm{~mL}$ of blood, which was carried out for preventing epithelial contamination. We lysed red blood cells within $72 \mathrm{~h}$, and we executed further negative selection using the EasySep Human CD45 Depletion Cocktail $(25 \mu \mathrm{L} / \mathrm{mL}$; STEMCELL Technologies Inc., Vancouver, BC, Canada) and EasySep Magnetic Nanoparticles $(50 \mu \mathrm{L} / \mathrm{mL}$; STEMCELL). Subsequently, the immunomagnetically enriched samples were subsequently spiked with OECM1/HCT116 cells, labelled using an Alexa Fluor ${ }^{\circ}$ 488-conjugated monoclonal antibody to EpCAM (1:400; Cell Signaling Technology Inc., Danvers, MA, USA), an Alexa Fluor 647 -conjugated monoclonal antibody to CD133 (1:200; CD133 [Novus Biologicals, Littleton, CO, USA]), and stained using a Hoechst 33342, blue fluorescent stain specific for DNA(20 mM; Thermo Fisher Scientific, USA). An isotype control antibody was used as an internal control, as well as the peripheral blood samples of healthy individuals $(4 \mathrm{~mL})$ that were and were not spiked with 1000 OECM1/HCT-166 cells, which were purchased from Taiwan's Food Industry Research and Development Institute. Performance recovery was defined as the proportion of OECM1/HCT-116 cells detected using flow cytometry (BD FACSCalibur; BD Biosciences, San Jose, CA, USA) to the number of spiked OECM1/HCT-116 cells, and a stable coefficient of variation $(\mathrm{CV})$ value has been calculated in previous studies $[32,33]$. In brief, the platform can have a recovery rate of $44.6 \pm 9.1 \%$ and a $\%$ coefficient of variation (CV) of $20.4 \%$. The previous platform reported in 2015 detected $13.1 \pm 0.9$ cells $/ \mathrm{mL}$ in healthy individuals $(n=20)$ [32], which was confusing and might be a background signal (i.e., a false positive result). In this revised platform, an isotype control was used for each sample, which resulted in a range of $0.0-3.0$ cells $/ \mathrm{mL}$ in healthy individuals in this study cohort $(\mathrm{n}=20)[32$, 33]. We defined CTCs as cells that tested positive for both EpCAM and Hoechst 33342. cCSCs were defined as cells expressing CD133, EpCAM and Hoechst simultaneously (Fig. 1).

\section{Statistical analysis}

For categorical and continuous variables, we report patients' demographic data as a number (\%) and median (range), respectively. We executed univariate and multivariate analyses; in the multivariate analysis, we examined all factors used in the executed univariate analysis, but we display only those factors with statistical significance. In addition, we analyzed the associations of variables with PFS and $\mathrm{OS}$ by employing a multivariate Cox proportional hazards model using a forward stepwise approach (logistic regression). We executed survival analysis by using the Kaplan-Meier method; the log-rank test was used to examine any differences. Optimal categorical (cut point) data analysis was performed using the X-tile statistical package (Yale University, New Haven, CT, USA) [34]. Correlations between CTCs or CCSCs and treatment response were calculated using the Pearson chi-squared or the Fisher exact test for an expected number of $<5$ per cell. We carried out the described statistical analyses with SPSS for Windows (version 18; SPSS Inc., Chicago, IL, USA). All executed statistical analyses in this study were two-sided. Moreover, we considered the observed differences to be significant when a $p$ value of $<0.05$ was obtained.

\section{Results}

Between April 2014 and January 2016, we prospectively enrolled 48 patients with metastatic breast cancer before they started receiving first-line palliative chemotherapy. Table 1 presents the baseline patient characteristics. The enrolled patients' median age was 52 years (range: 28 81 years); all the patients were women $(100.0 \%)$. The performance status, determined on the basis of the Eastern Cooperative Oncology Group (ECOG) guideline was between 0 and 1 for most enrolled patients (85.4\%). According to the 7th Edition AJCC criteria, all patients had stage IV disease. Thirty-five patients $(72.9 \%)$ were ER and PR, and 23 (47.9\%) were positive for HER-2. Eight patients (16.7\%) had triple-negative breast cancer. Thirty-two patients $(66.7 \%)$ had more than two metastatic sites. The bones were the most common metastatic site $(72.9 \%)$, followed by the liver (37.5\%), distant lymph nodes (35.4\%), lung (33.3\%), brain (14.6\%), and pleura (12.5\%). Thirty-four patients $(70.8 \%)$ had visceral metastases. A depiction of cCSCs and CTCs is presented in Fig. 1. We identified CTCs in all the enrolled patients, with the median number of identified CTCs being 33.9/ $\mathrm{mL}$ (range: $4.5-555 / \mathrm{mL}$ ). We isolated cCSCs in $97.9 \%$ of 


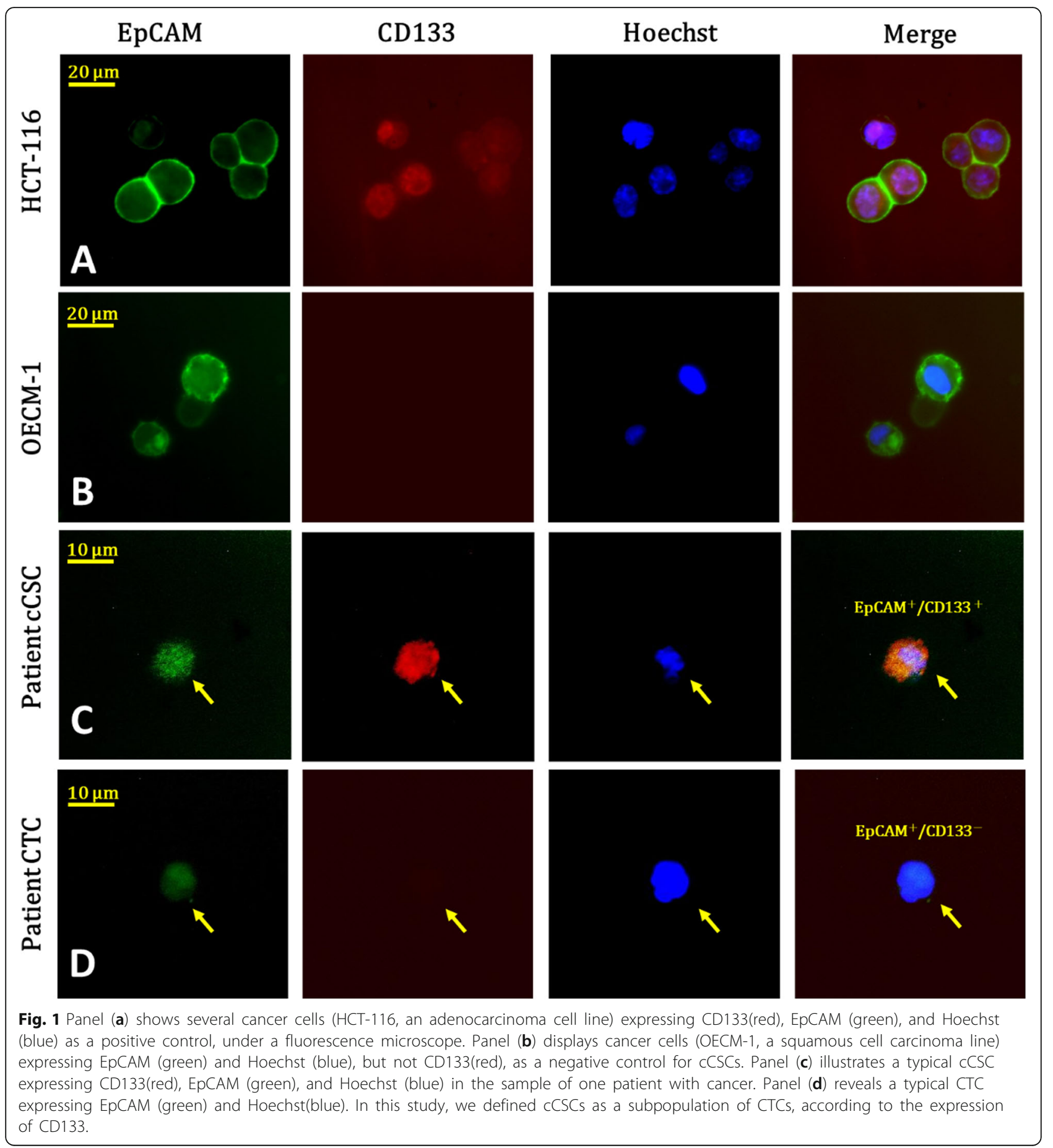

patients, and the median proportion of CSCs was $14.7 \%$. After a median duration of follow-up of 24.7 months (range, 0.6-43.5 months), we observed that of the 48 patients enrolled in the study, $43(89.5 \%)$ had disease progression and 16 (33.3\%) died. The median duration of PFS was 8.5 months (95\% confidence interval: $5.3-11.7$ months), and the median OS had not been reached by the end of the study.
According to a receiver operating characteristic analysis, the optimal cutoffs for high and low measurement values of CTCs and CSCs were $13.3 / \mathrm{mL}$ and $32.5 \%$, respectively. No statistically significant difference was observed between the group with low CTCs and that with high CTCs regarding the chemotherapy response rate (79.2\% vs. $70.8 \%, p=0.505)$ after 3 months of treatment (Fig. 2A) and progression-free survival (PFS; 8.5 months 
Table 1 Basic characteristics of enrolled patients $(N=48)$

\begin{tabular}{|c|c|c|}
\hline & $\mathrm{N}$ & $\%$ \\
\hline Age, median, years (range) & $52(28-81)$ & \\
\hline \multicolumn{3}{|l|}{ Sex } \\
\hline Female & 48 & $100.0 \%$ \\
\hline \multicolumn{3}{|l|}{ Staging (AJCC 7th Edition) } \\
\hline Stage IV & 48 & $100.0 \%$ \\
\hline \multicolumn{3}{|l|}{ Performance status (ECOG) } \\
\hline $0-1$ & 41 & $85.4 \%$ \\
\hline$\geq 2$ & 7 & $14.6 \%$ \\
\hline \multicolumn{3}{|l|}{ Receptor status } \\
\hline ER and/or PR positive & 35 & $72.9 \%$ \\
\hline HER-2/neu positive & 23 & $47.9 \%$ \\
\hline Triple-negative (ER/PR/HER2) & 8 & $16.7 \%$ \\
\hline \multicolumn{3}{|l|}{ Number of metastases } \\
\hline Single metastasis & 16 & $33.3 \%$ \\
\hline$\geq 2$ metastases & 32 & $66.7 \%$ \\
\hline \multicolumn{3}{|c|}{ Site of distant metastasis at study enrollment } \\
\hline Bone & 35 & $72.9 \%$ \\
\hline Liver & 18 & $37.5 \%$ \\
\hline Distant lymph nodes & 17 & $35.4 \%$ \\
\hline Lung & 16 & $33.3 \%$ \\
\hline Brain & 7 & $14.6 \%$ \\
\hline Pleura & 6 & $12.5 \%$ \\
\hline Visceral metastasist & 34 & $70.8 \%$ \\
\hline Nonvisceral metastasis & 14 & $29.2 \%$ \\
\hline
\end{tabular}

Abbreviations: AJCC, American Joint Committee on Cancer; ECOG, Eastern Cooperative Oncology Group. SD: standard deviation; Cl: confidence interval. $E R$, estrogen receptor; PR, progesterone receptor; HER2, human epidermal growth factor receptor 2; PFS: progression-free survival; OS: overall survival †Visceral sites include the lungs, liver, brain, adrenal glands, and pleura (with or without effusion). Nonvisceral sites were defined as the breast, lymph nodes, chest wall, bones, and skin

vs. 8.2 months, $p=0.862$; Fig. 3A). Patients with low CTCs counts had a better overall survival (OS) than did those with high CTCs counts (18.9 months vs. 5.7 months, $p<$ 0.001 ; Fig. 4A). However, a low cCSCs count was correlated with a superior tumor response rate (95.8\% vs. $54.2 \%, \mathrm{p}<0.001$; Fig. 2B), PFS (median, 18.0 vs. 5.7 months, $\mathrm{p}<0.001$; Fig. $3 \mathrm{~B}$ ), and OS (median, not reached vs. 27.7 months, $\mathrm{p}<0.001$; Fig. $4 \mathrm{~B}$ ), compared with a high cCSCs count. In combination with other clinical parameters, a multivariate analysis showed that ECOG performance status, triple-negative (ER/PR/HER2) type, and baseline cCSCs were independent prognostic factors for PFS. Baseline CTCs and cCSCs were independent prognostic factors for OS (Table 2).

\section{Discussion}

On the basis of evidence indicating that CD133 is a stem cell marker [12], we hypothesized that cCSCs, which are
CD133-expressing CTCs, have a role in chemotherapy resistance and breast cancer prognosis. We therefore performed the current prospective multicenter study that evaluated 48 patients with unresectable and/or metastatic stage IV breast cancer undergoing first-line palliative chemotherapy. We revealed several elementary but crucial findings in terms of the function of CTCs as well as cCSCs in metastatic breast cancer. We demonstrated that patients with high baseline cCSCs before chemotherapy had inferior outcomes in terms of chemotherapy response, PFS, and OS. High baseline CTCs also predicted less favorable OS, but this was not associated with chemotherapy response or PFS. In the multivariate analysis, poor performance status, triple-negative type, and baseline cCSCs were independent prognostic factors for PFS. However, baseline CTCs and CSCs were independent predictors of OS.

The CTC quantity has emerged as a potential biomarker, and the presence of CTCs in peripheral blood was reported to be correlated with a relatively poor prognosis of metastatic breast cancer [35]. In the current study, pretreatment CTCs remained an independent prognostic factor for OS, a finding that was in line with those of related studies [3537]. However, in the current study, performance status, triple-negative type, and baseline cCSCs, rather than CTCs, were independent prognostic factors for PFS, which partly contradicts the findings of relevant studies [35-38]. Some plausible reasons why the CTC number influences OS rather than the first PFS include (i) the limited sample size (ii) the fact that chemotherapy regimens were not the same in the entire cohort; and (iii) breast cancer patients in realworld settings would possibly receive multiple combination treatments, such as local palliative radiotherapy, which might alter the first PFS but not OS in this cohort. The most useful prognostic factors in clinical settings for breast cancer are the performance status, age, site of metastasis (presence of visceral metastasis or not), and HR and HER2 status [39, 40]. Patients having a poor performance status may not tolerate intensive chemotherapy, and such patients may experience high toxicity during chemotherapy [41]. Of the patients included in the current study, $14.6 \%$ had a relatively poor performance status $(\geq 2)$. Clinically, it is not surprising that a poor performance status is associated with poor PFS, which was also demonstrated in our study. Nevertheless, the prognosis of patients diagnosed as having triple-negative breast cancer was reported to be poorer than that of those diagnosed as having other subtypes of breast cancer [42], which is also in accordance with our study results. However, in addition to the clinical prognostic factors, baseline cCSCs remained an independent prognostic factor for OS and PFS, suggesting a more powerful role of this subset of CTCs.

Research has analyzed the role of cCSCs in many cancers, which have been examined using RT-PCR [27, 43], 


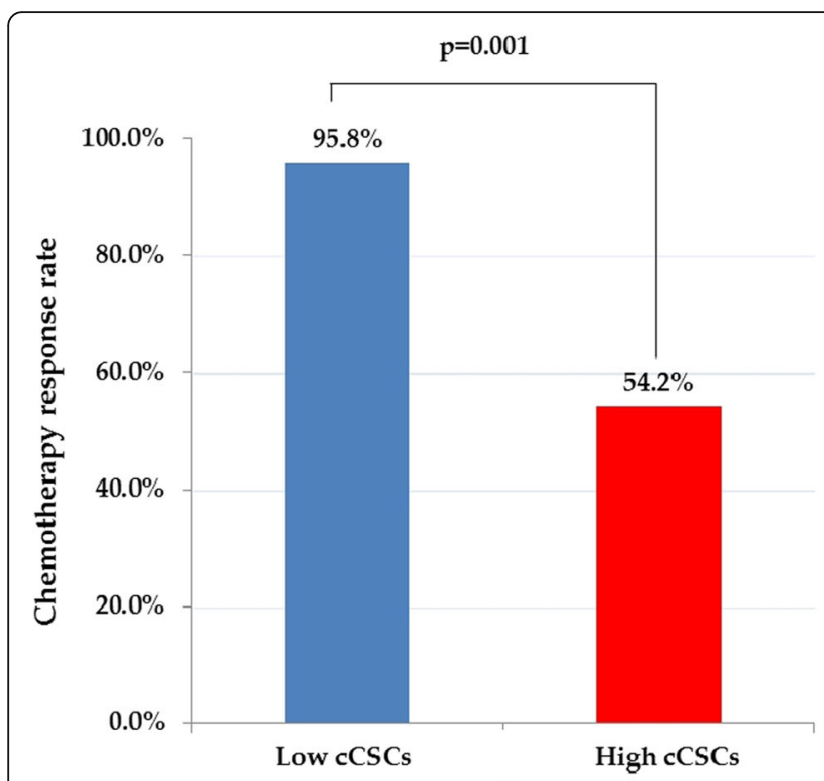

A

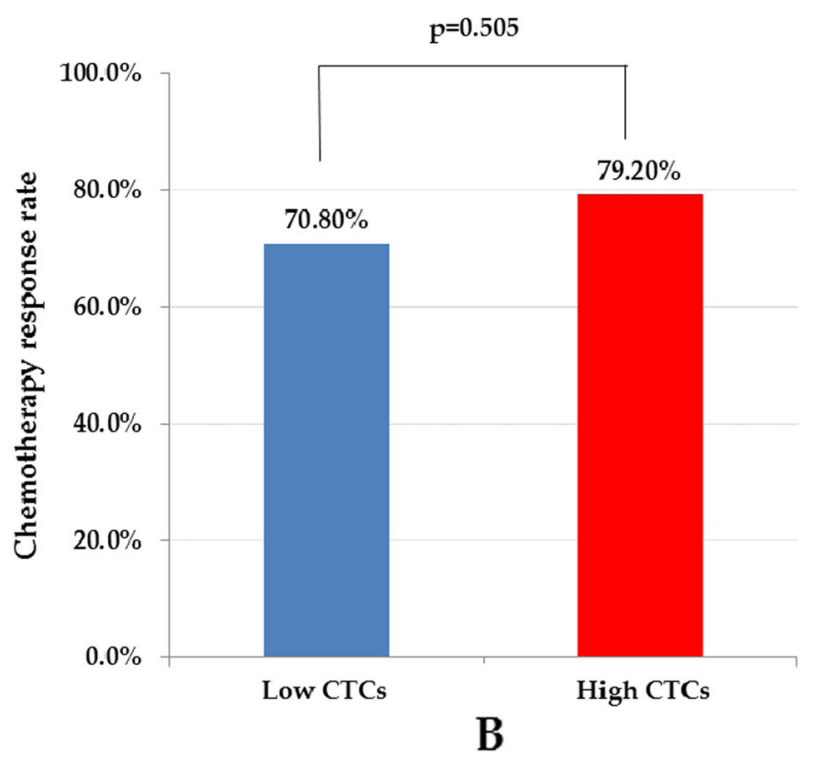

Fig. 2 Chemotherapy response rate stratified by baseline (A) circulating tumor cells (CTCS) and (B) circulating cancer stem-like cells (cCSCs)

enumeration with a gradient protocol [44, 45], and flow cytometry $[46,47]$. The results of such research have indicated that $\mathrm{CCSCs}$ are linked to an unfavorable prognosis in various cancers, including colorectal cancer [27, 48], gastric cancer [45], lung cancer [46], prostate cancer [49], head and neck cancer [50] and hepatocellular carcinoma [43]. Nadal et al. [12] revealed the detection of a relative enrichment of CD133-expressing cells in nonluminal tumor subtype nonmetastatic breast cancer after chemotherapy, indicating a possible role of CD133expressing CTCs in processes of chemotherapy resistance; however, those authors did not analyze the prognostic role of such CTCs. According to our review of the relevant literature, our research is the first



A

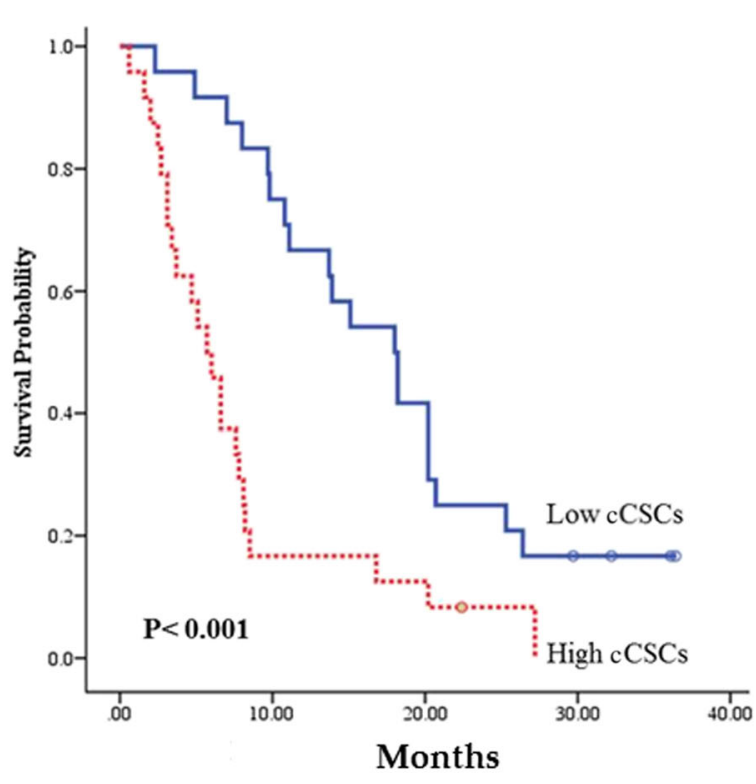

B

Fig. 3 (A) Comparison of progression-free survival (PFS; months) between patients with a high circulating tumor cells (CTCs) counts (dotted line) and low CTCs counts (solid line). (B) Comparison of PFS (months) between patients with high circulating cancer stem-like cells (cCSCs) counts (dotted line) and low cCSCs counts (solid line). Censored observations are indicated as points on the curves 


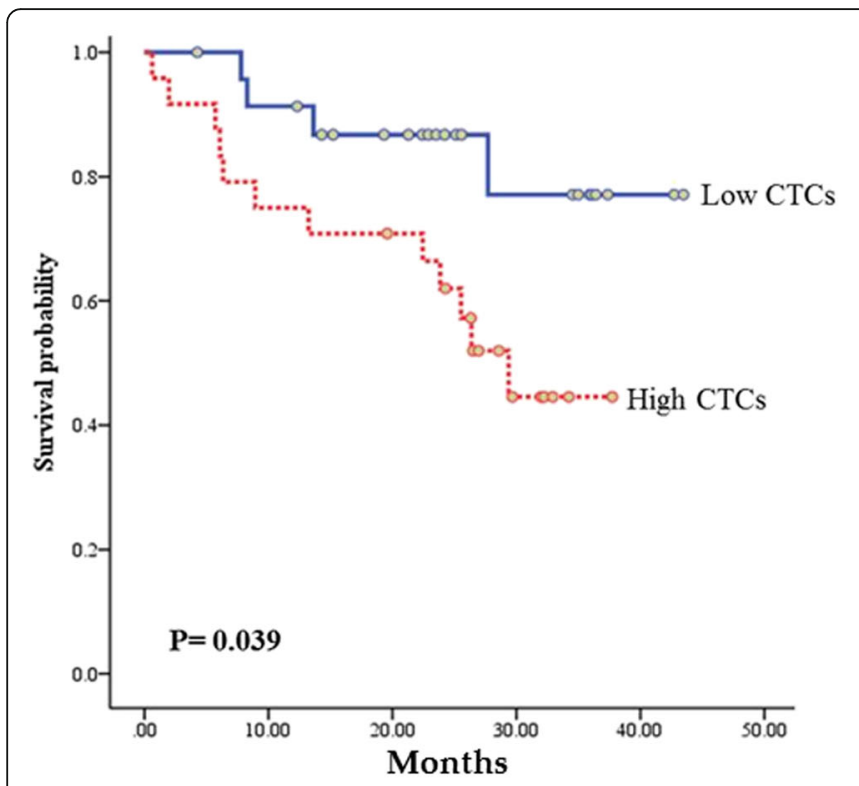

A

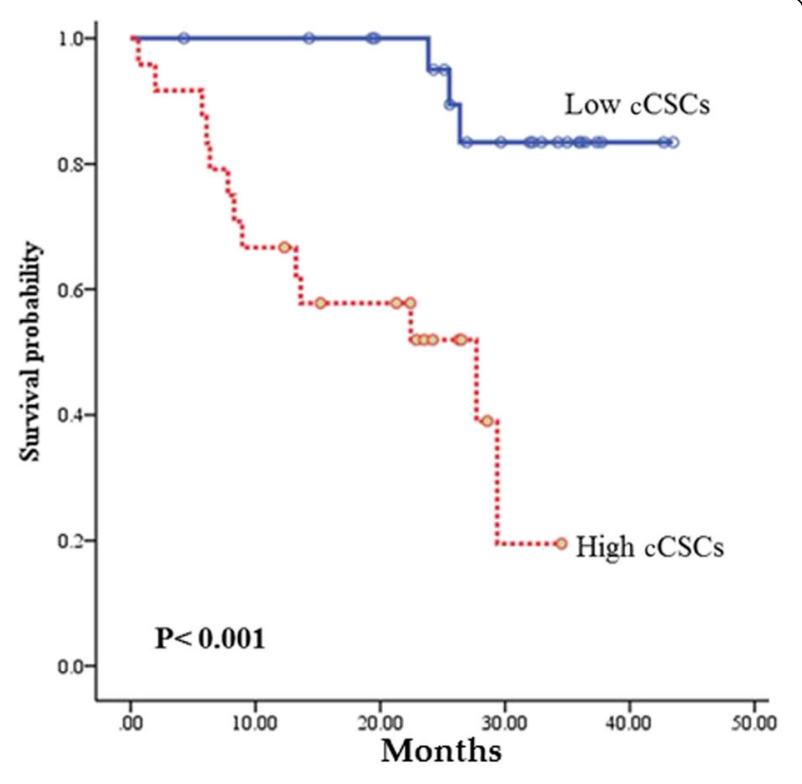

B

Fig. 4 (A) Comparison of overall survival (OS; months) between patients with high circulating tumor cell (CTCs) counts (dotted line) and low CTCS counts (solid line). (B) Comparison of OS (months) between patients with high circulating cancer stem-like cell (cCSCs) counts (dotted line) and low cCSCs counts (solid line). Censored observations are indicated as points on the curves

prospective study to examine the prognostic significance using a single blood sample that was obtained from patients having metastatic breast cancer undergoing firstline chemotherapy, showing that baseline cCSCs is an independent prognostic factor for survival, in combination with the other clinical prognostic factors.

A reliable method developed for detecting or isolating CTCs could act as a valuable predictor before anticancer treatment is administered in patients with cancer. Although a previously developed CellSearch ${ }^{\circ}$ system was granted approval by the US Food and Drug Administration (FDA) in 2004, it is unavailable in most hospitals. To date, no standard protocol or method is available for identifying or isolating CTCs. This is largely owing to the relatively low detection efficiency and high cost of each test. In 2015, our team developed a negative selection strategy combined with flow cytometry for the identification of CTCs with multiple surface marker expressions, which has received validation for numerous cancer types [14, 16, 32]. Compared with the only FDAapproved device, CellSearch ${ }^{\circ}$, the method used in this study has demonstrated a similar recovery rate and better detection rate in late-stage cancer settings [35]. Crucially, CTC testing based on the negative selection strategy has a greater likelihood of preserving cells without cytokeratins or EpCAM expression [8, 51, 52]. These cells should be preserved during isolation processes, and the negative selection protocol offers the most favorable

Table 2 Univariate and multivariate analyses for progression-free and overall survival

\begin{tabular}{|c|c|c|c|c|c|c|c|c|}
\hline \multirow{3}{*}{ Parameters } & \multicolumn{4}{|l|}{ PFS } & \multicolumn{4}{|l|}{ OS } \\
\hline & \multicolumn{2}{|l|}{ Univariate } & \multicolumn{2}{|l|}{ Multivariate } & \multicolumn{2}{|l|}{ Univariate } & \multicolumn{2}{|l|}{ Multivariate } \\
\hline & HR $(95 \% \mathrm{Cl})$ & $\mathrm{p}$ & HR $(95 \% \mathrm{Cl})$ & $p$ & HR $(95 \% \mathrm{Cl})$ & $p$ & HR $(95 \% \mathrm{Cl})$ & $\mathrm{p}$ \\
\hline Age $>65 y$ & $1.007(0.983-1.032)$ & 0.584 & & & $1.001(0.960-1.004)$ & 0.968 & & \\
\hline ECOG PS $>1$ & $4.441(1.948-10.127)$ & $<0.001$ & $3.544(1.413-8.889)$ & 0.005 & $3.390(1.161-9.898)$ & 0.026 & & \\
\hline Visceral metastasist & $1.410(0.723-2.752)$ & 0.314 & & & $3.473(0.785-15.386)$ & 0.101 & & \\
\hline $\begin{array}{l}\text { Triple-negative } \\
\text { (ER/PR/HER2) }\end{array}$ & $4.463(1.900-10.483)$ & 0.001 & $3.329(1.328-8.342)$ & 0.010 & $3.995(1.177-13.296)$ & 0.026 & & \\
\hline $\mathrm{CTCs}>32 / \mathrm{ml}$ & $1.003(1.000-1.006)$ & 0.089 & & & $1.006(1.003-1.010)$ & $<0.001$ & $7.266(1.775-29.741)$ & 0.006 \\
\hline $\operatorname{cCSC}>15 \%$ & $2.903(1.563-5.392)$ & 0.001 & $2.867(1.338-6.140)$ & $<0.0001$ & $8.236(2.269-29.894)$ & 0.001 & $16.238(3.167-83.264)$ & 0.001 \\
\hline
\end{tabular}

Abbreviations: OS: overall survival; PFS: progression-free survival; HR: hazard ratio; ECOG PS: Eastern Cooperative Oncology Group performance wtatus; ER, estrogen receptor; PR, progesterone receptor; HER2, human epidermal growth factor receptor 2; CTCs: circulating tumor cells; cCSCs: circulating cancer stem cells 
opportunity for this. In the current study, we detected CTCs in all patients and isolated cCSCs in $97.9 \%$ of patients, indicating a high detection rate. Our platform provides a superior detection rate and might have more success in identifying more CTCs, to determine a cutoff of clinical importance. The cutoff value for the number of CTCs and cCSCs determined using receiver operating characteristic curves was also analyzed as an independent prognostic factor for survival. On the basis of this straightforward and inexpensive method, we posit that analyzing the CTC and CCSC ratios through flow cytometry combined with a negative selection strategy, as done in the current study, represents a widely available method that can be used to determine predictive and prognostic factors in patients undergoing first-line palliative chemotherapy for metastatic breast cancer. The methodology of CTC detection still requires standardization and an automatic device, to maintain a stable CV. The method used in the current study involves operator-dependent analysis, although this approach could be applied in a small laboratory, such as those in most hospitals. Consensus on the standardization of protocols is still required.

Certain limitations should be acknowledged when evaluating this study. First, the study had a small number of participants, which limits the strength of our results. However, estimating an ideal sample size in a biomarker study is difficult; also, the sensitivity of the tool used in the current study had not been reported or tested in the same population previously. The lack of sensitivity highlights the limitation of the sample size estimation. Therefore, this study should be viewed as an exploratory study, even though our results are statistically significant. Second, the chemotherapy regimen was inconsistent among patients; therefore, the analysis of the baseline CTCs and CSCs associated with survival might have been confounded by different treatment regimens. Third, although we used a clear and strict definition of cCSCs, we did not isolate these cells to confirm that they possessed tumorigenicity in vivo, invasion, or self-renewal, which is why we refer to them as stem-like cells. Third, we did not include cytokeratins (CK) as one of the markers given the fact that fixation and permeabilization processes commonly lose cells, which is the main goal we want to avoid in the negative selection strategy. Fourth, we did not perform CD45 characterization in the downstream analysis, which might cause some bias in CTC identification. Therefore, larger independent trials are required to validate our findings.

\section{Conclusions}

We identified cCSCs at baseline palliative chemotherapy as an independent prognostic factor for predicting survival outcomes such as OS, and PFS, as well as tumor response in patients diagnosed as with metastatic breast cancer. We believe that these findings facilitate the identification of patients with the least favorable prognoses and treatment outcomes in clinical settings.

\section{Supplementary information}

Supplementary information accompanies this paper at https://doi.org/10. 1186/s12885-019-6370-1.

Additional File 1. Correlations among baseline patient characteristics and baseline CCSC ratio. Abbreviations: CCSCs: circulating cancer stem cells; AJCC, American Joint Committee on Cancer; ECOG, Eastern Cooperative Oncology Group; SD: standard deviation; Cl: confidence interval; HR, hormone receptor; HER2, human epidermal growth factor receptor 2; PFS: progression-free survival; OS: overall survival. +Visceral sites include the lungs, liver, brain, adrenal glands, and pleura (with or without effusion). Nonvisceral sites were defined as the breast, lymph nodes, chest wall, bones, and skin.

\section{Abbreviation}

cCSCs: Circulating cancer stem-like cells; CD: Cell surface; CECs: Circulating epithelial cells; CT: Computed tomography; CTCs: Circulating tumor cells; ECOG: Eastern Cooperative Oncology Group; EpCAM: Epithelial cell adhesion molecule; ERs: Estrogen receptors; HER-2: Human epidermal growth factor receptor 2; HR: Hormone receptors; OS: Overall survival; PFS: Progression-free survival; PR: Progesterone receptors

\section{Acknowledgments}

The authors thank all members of the Cancer Centre, Chang Gung Memorial Hospital, for their invaluable help. We also gratefully acknowledge the reviewer's invaluable advice.

\section{Author's contribution}

$\mathrm{CHL}, J \mathrm{CHH}$, and PHC conceptualized the study. JCHH, TSY, YCL, JSC, CLL, and WCC curated the data. MHW analyzed the data. $\mathrm{CHL}, \mathrm{JCCH}, \mathrm{HMW}$, and PHC obtained the funding for the study. TSY performed the study investigation. $\mathrm{PHC}$ was the project administrator. MHW, HHW, WKH, TMH, TTY, and SCC provided the required resources. SCC provided the software. YCL, JSC, and TTY supervised the study. CLL, WKH, TMH, WCC, FCK, and CCH validated the study findings. CLL wrote the original draft of the manuscript. FCK and PHC rewrote the manuscript considering the reviewer's comments. All authors have read and approved the final manuscript.

\section{Funding}

This research was funded by Chang Gung Memorial Hospital, CMRPG2D0171, CMRPG2D0172, CMRPG2G0681,

CMRPG2G0682, CMRPG2G0683, CMRPG2J0061-3 to PH Chang;

CMRPG2D0173 to Chun-Hui Lee, PMRPG3H0071, CMRPG3G0593,

CORPG3G0791, CORPG3G0771, CMRPG3E1631-33, MOST-108-2628-B-182A001 to CH Hsieh; CMRPG3G1131-1133, CMRPG3H0871-73, MOST-107-2314-B182-053, MOST-104-2314-B-182-031-MY3 to HM Wang. The funding source had no role in the design of the study and collection, analysis, interpretation of data, or in writing the manuscript.

\section{Availability of data and materials}

The datasets used or analyzed during the current study are available from the corresponding author on reasonable request.

\section{Ethics approval and consent to participate}

The study protocol was approved by the Institutional Review Board of Chang Gung Memorial Hospital (approval ID: 103-0425B and 103-5322B). For protocols that required ethical approval, written informed consent was obtained from all patients.

Consent for publication

Not applicable.

Competing interests

The authors declare that they have no competing interest. 


\section{Author details}

${ }^{1}$ Division of General Surgery, Department of Surgery, Chang Gung Memorial Hospital, Keelung, Taiwan. ${ }^{2}$ Circulating Tumour Cell Lab, Division of Medical Oncology, Department of Internal Medicine, Chang Gung Memorial Hospital at Linkou, Taoyuan, Taiwan. ${ }^{3}$ College of Medicine, Chang Gung University, Taoyuan, Taiwan. ${ }^{4}$ Department of Chemical and Materials Engineering, Chang Gung University, Taoyuan, Taiwan. ${ }^{5}$ Graduate Institute of Biomedical Engineering, Chang Gung University, Taoyuan City 33302, Taiwan. ${ }^{6}$ Department of Chemical Engineering, Ming Chi University of Technology, New Taipei City 24301, Taiwan. ${ }^{7}$ Division of Endocrinology and Metabolism, Department of Internal Medicine, Taichung Veterans General Hospital, Taichung, Taiwan. ${ }^{8}$ Department of Public Health, College of Public Health, China Medical University, Taichung, Taiwan. ${ }^{9}$ Department of Medical Research, Taichung Veterans General Hospital, Taichung, Taiwan. ${ }^{10}$ Department of Oncology-Pathology, Karolinska Institutet, Stockholm, Sweden, Cancer Center Karolinska, Karolinska University Hospital, SE-17176 Stockholm, Sweden. ${ }^{11}$ Department of Radiation Oncology, Chang Gung Memorial Hospital, College of Medicine, Chang Gung University, Taoyuan, Taiwan. ${ }^{12}$ Nuclear Medicine and Molecular Imaging Center, Linkou Chang Gung Memorial Hospital, Taoyuan, Taiwan. ${ }^{13}$ Department of Hematology and Oncology, Department of Medicine, Chang-Gung Memorial Hospital, Chiayi 61363, Taiwan. ${ }^{14}$ Department of Hepatogastroenterology, Chang Gung Memorial Hospital, Keelung, Taiwan. ${ }^{15}$ Division of Hematology-Oncology, Department of Internal Medicine, Chang Gung Memorial Hospital, Keelung, Taiwan.

Received: 8 February 2019 Accepted: 18 November 2019 Published online: 02 December 2019

\section{References}

1. Siegel RL, Miller KD, Jemal A. Cancer statistics, 2018. CA Cancer J Clin. 2018; 68:7-30.

2. Largillier R, Ferrero JM, Doyen J, Barriere J, Namer M, Mari V, et al. Prognostic factors in 1,038 women with metastatic breast cancer. Ann Oncol. 2008;19:2012-9.

3. Li SR, Zhao JL, Zhu LL, Su FX, Chen K. Development and validation of a nomogram predicting the overall survival of stage IV breast cancer patients. Cancer Medicine. 2017;6:2586-94.

4. Tong CWS, Wu M, Cho WCS, To KKW. Recent advances in the treatment of breast cancer. Front Oncol. 2018;8:227.

5. Hattori M, Iwata $\mathrm{H}$. Advances in treatment and care in metastatic breast cancer $(\mathrm{MBC})$ : are there $\mathrm{MBC}$ patients who are curable? Chin Clin Oncol. 2018;7:23.

6. Alunni-Fabbroni M, Müller V, Fehm T, Janni W, Rack B. Monitoring in metastatic breast cancer: is imaging outdated in the era of circulating tumor cells? Breast Care. 2014;9:16-21.

7. Ashworth T. A case of cancer in which cells similar to those in the tumours were seen in the blood after death. Aust Med J. 1869;14:146.

8. de Wit S, van Dalum G, Lenferink AT, Tibbe AG, Hiltermann TJ, Groen HJ, et al. The detection of EpCAM(+) and $\operatorname{EpCAM(-)~circulating~tumor~cells.~Sci~}$ Rep. 2015;5:12270.

9. Jiang J, Zhao H, Shu W, Tian J, Huang Y, Song Y, et al. An integrated microfluidic device for rapid and high-sensitivity analysis of circulating tumor cells. Sci Rep. 2017;7:42612.

10. Lapin M, Tjensvoll K, Oltedal S, Buhl T, Gilje B, Smaaland R, et al. MINDEC-an enhanced negative depletion strategy for circulating tumour cell enrichment. Sci Rep. 2016;6:28929.

11. Yu M, Bardia A, Aceto N, Bersani F, Madden MW, Donaldson MC, et al. Cancer therapy. Ex vivo culture of circulating breast tumor cells for individualized testing of drug susceptibility. Science. 2014;345:216-20.

12. Nadal R, Ortega FG, Salido M, Lorente JA, Rodriguez-Rivera M, DelgadoRodriguez M, et al. CD133 expression in circulating tumor cells from breast cancer patients: potential role in resistance to chemotherapy. Int J Cancer. 2013;133:2398-407.

13. Kenneth HY, Ricigliano M, Hidalgo M, Abou-Alfa GK, Lowery MA, Saltz LB, et al. Pharmacogenomic modeling of circulating tumor and invasive cells for prediction of chemotherapy response and resistance in pancreatic cancer. Clin Cancer Res. 2014;20:5281-9.

14. Chou W-C, Wu M-H, Chang P-H, Hsu H-C, Chang G-j, Huang W-k, et al. A prognostic model based on circulating tumour cells is useful for identifying the poorest survival outcome in patients with metastatic colorectal cancer. Int J Biol Sci. 2018;14:137.

15. Krebs MG, Sloane R, Priest L, Lancashire L, Hou J-M, Greystoke A, et al. Evaluation and prognostic significance of circulating tumor cells in patients with non-small-cell lung cancer. J Clin Oncol. 2011;29(12):1556-63.

16. Hsieh $\mathrm{JCH}$, Lin $\mathrm{HC}$, Huang $\mathrm{CY}$, Hsu HL, Wu TMH, Lee $\mathrm{CL}$, et al. Prognostic value of circulating tumor cells with podoplanin expression in patients with locally advanced or metastatic head and neck squamous cell carcinoma. Head Neck. 2015:37:1448-55.

17. Cristofanilli M, Budd GT, Ellis MJ, Stopeck A, Matera J, Miller MC, et al. Circulating tumor cells, disease progression, and survival in metastatic breast cancer. N Engl JMed. 2004;351:781-91.

18. Todaro M, Francipane MG, Medema JP, Stassi G. Colon cancer stem cells: promise of targeted therapy. Gastroenterology. 2010;138:2151-62.

19. Aktas B, Tewes M, Fehm T, Hauch S, Kimmig R, Kasimir-Bauer S. Stem cell and epithelial-mesenchymal transition markers are frequently overexpressed in circulating tumor cells of metastatic breast cancer patients. Breast Cancer Res. 2009;11:R46.

20. Zeisberg M, Neilson EG. Biomarkers for epithelial-mesenchymal transitions. J Clin Invest. 2009;119:1429-37.

21. Theodoropoulos PA, Polioudaki H, Agelaki S, Kallergi G, Saridaki Z, Mavroudis D, et al. Circulating tumor cells with a putative stem cell phenotype in peripheral blood of patients with breast cancer. Cancer Lett. 2010;288:99-106.

22. Kasimir-Bauer S, Hoffmann O, Wallwiener D, Kimmig R, Fehm T. Expression of stem cell and epithelial-mesenchymal transition markers in primary breast cancer patients with circulating tumor cells. Breast Cancer Res. 2012;14:R15.

23. Yang M-H, Imrali A, Heeschen C. Circulating cancer stem cells: the importance to select. Chin J Cancer Res. 2015;27:437.

24. Vander Griend DJ, Karthaus WL, Dalrymple S, Meeker A, DeMarzo AM, Isaacs JT. The role of CD133 in normal human prostate stem cells and malignant cancer-initiating cells. Cancer Res. 2008;68:9703-11.

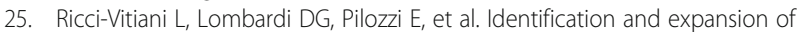
human colon-cancer-initiating cells. Nature. 2007:445:111-5.

26. Liou GY. CD133 as a regulator of cancer metastasis through the cancer stem cells. Int J Biochem Cell Biol. 2018;106:1-7.

27. linuma H, Watanabe T, Mimori K, Adachi M, Hayashi N, Tamura J, et al, Clinical significance of circulating tumor cells, including cancer stem-like cells, in peripheral blood for recurrence and prognosis in patients with Dukes' stage B and C colorectal cancer. J Clin Oncol. 2011;29:1547-55.

28. Abdallah EA, Fanelli MF, Buim ME, Machado Netto MC, Gasparini Junior JL, Souza ESV, et al. Thymidylate synthase expression in circulating tumor cells: a new tool to predict 5-fluorouracil resistance in metastatic colorectal cancer patients. Int J Cancer. 2015;137:1397-405.

29. Paoletti C, Cani AK, Larios JM, Hovelson DH, Aung K, Darga EP, et al. Comprehensive mutation and copy number profiling in archived circulating breast cancer tumor cells documents heterogeneous resistance mechanisms. Cancer Res. 2018;78:1110-22.

30. Mego M, Mani SA, Lee BN, Li C, Evans KW, Cohen EN, et al. Expression of epithelial-mesenchymal transition-inducing transcription factors in primary breast cancer: the effect of neoadjuvant therapy. Int J Cancer. 2012;130:808-16.

31. Eisenhauer EA, Therasse P, Bogaerts J, Schwartz LH, Sargent D, Ford R, et al. New response evaluation criteria in solid tumours: revised RECIST guideline (version 1.1). Eur J Cancer. 2009:45:228-47.

32. Su PJ, Wu MH, Wang HM, Lee CL, Huang WK, Wu CE, et al. Circulating tumour cells as an independent prognostic factor in patients with advanced oesophageal squamous cell carcinoma undergoing chemoradiotherapy. Sci Rep. 2016;6:31423.

33. Chiu TK, Chou WP, Huang SB, Wang HM, Lin YC, Hsieh CH, et al. Application of optically-induced-dielectrophoresis in microfluidic system for purification of circulating tumour cells for gene expression analysis- Cancer cell line model. Sci Rep. 2016;6:32851.

34. Camp RL, Dolled-Filhart M, Rimm DL. X-tile: a new bio-informatics tool for biomarker assessment and outcome-based cutpoint optimization. Clin Cancer Res. 2004;10:7252-9.

35. Bidard FC, Peeters DJ, Fehm T, Nole F, Gisbert-Criado R, Mavroudis D, et al. Clinical validity of circulating tumour cells in patients with metastatic breast cancer: a pooled analysis of individual patient data. Lancet Oncol. 2014;15:406-14.

36. Cristofanilli M, Hayes DF, Budd GT, Ellis MJ, Stopeck A, Reuben JM, et al. Circulating tumor cells: a novel prognostic factor for newly diagnosed metastatic breast cancer. J Clin Oncol. 2005;23:1420-30.

37. Hayes DF, Cristofanilli M, Budd GT, Ellis MJ, Stopeck A, Miller MC, et al. Circulating tumor cells at each follow-up time point during therapy of 
metastatic breast cancer patients predict progression-free and overall survival. Clin Cancer Res. 2006;12:4218-24.

38. Cristofanilli M, Pierga J-Y, Reuben J, Rademaker A, Davis AA, Peeters DJ, et al. The clinical use of circulating tumor cells (CTCS) enumeration for staging of metastatic breast cancer $(\mathrm{MBC})$ : international expert consensus paper. Crit Rev Oncol Hematol. 2018;134:39-45.

39. Barrios CH, Sampaio C, Vinholes J, Caponero R. What is the role of chemotherapy in estrogen receptor-positive, advanced breast cancer? Ann Oncol. 2009;20:1157-62.

40. Swenerton KD, Legha SS, Smith T, Hortobagyi GN, Gehan EA, Yap HY, et al. Prognostic factors in metastatic breast cancer treated with combination chemotherapy. Cancer Res. 1979;39:1552-62.

41. Clark GM, Sledge GW Jr, Osborne CK, McGuire WL. Survival from first recurrence: relative importance of prognostic factors in 1,015 breast cancer patients. J Clin Oncol. 1987:5:55-61.

42. Ismail-Khan R, Bui MM. A review of triple-negative breast cancer. Cancer Control. 2010;17:173-6.

43. Bahnassy AA, Zekri AR, El-Bastawisy A, Fawzy A, Shetta M, Hussein N, et al. Circulating tumor and cancer stem cells in hepatitis $C$ virus-associated liver disease. World J Gastroenterol. 2014;20:18240-8.

44. Malara N, Trunzo V, Foresta U, Amodio N, De Vitis S, Roveda L, et al. Ex-vivo characterization of circulating colon cancer cells distinguished in stem and differentiated subset provides useful biomarker for personalized metastatic risk assessment. J Transl Med. 2016;14:133.

45. Toyoshima K, Hayashi A, Kashiwagi M, Hayashi N, Iwatsuki M, Ishimoto T, et al. Analysis of circulating tumor cells derived from advanced gastric cancer. Int J Cancer. 2015;137:991-8.

46. Pirozzi G, Tirino V, Camerlingo R, La Rocca A, Martucci N, Scognamiglio G, et al. Prognostic value of cancer stem cells, epithelial-mesenchymal transition and circulating tumor cells in lung cancer. Oncol Rep. 2013;29:1763-8.

47. Skirecki T, Hoser G, Kawiak J, Dziedzic D, Domagala-Kulawik J. Flow cytometric analysis of CD133- and EpCAM-positive cells in the peripheral blood of patients with lung cancer. Arch Immunol Ther Exp. 2014;62:67-75.

48. Park BS, Jung SY, Kwon SM, Bae JH, Lee SM, Shin DH, et al. Comparison of putative circulating cancer stem cell detection between the hepatic portal system and peripheral blood in colorectal cancer patients. Ann Surg Treat Res. 2014:87:232-8.

49. Rentala S, Chintala R, Guda M, Chintala M, Komarraju AL, Mangamoori LN. Atorvastatin inhibited rho-associated kinase 1 (ROCK1) and focal adhesion kinase (FAK) mediated adhesion and differentiation of CD133+CD44+ prostate cancer stem cells. Biochem Biophys Res Commun. 2013;441:586-92.

50. Chang PH, Wu MH, Liu SY, Wang HM, Huang WK, Liao CT, et al. The prognostic roles of pretreatment circulating tumor cells, circulating cancer stem-like cells, and programmed cell death-1 expression on peripheral lymphocytes in patients with initially unresectable, recurrent or metastatic head and neck cancer: an exploratory study of three biomarkers in onetime blood drawing. Cancers. 2019;11:540.

51. Satelli A, Batth IS, Brownlee Z, Rojas C, Meng QH, Kopetz S, et al. Potential role of nuclear PD-L1 expression in cell-surface vimentin positive circulating tumor cells as a prognostic marker in cancer patients. Sci Rep. 2016;6:28910.

52. Grover PK, Cummins AG, Price TJ, Roberts-Thomson IC, Hardingham JE. Circulating tumour cells: the evolving concept and the inadequacy of their enrichment by EpCAM-based methodology for basic and clinical cancer research. Ann Oncol. 2014;25:1506-16.

\section{Publisher's Note}

Springer Nature remains neutral with regard to jurisdictional claims in published maps and institutional affiliations.

Ready to submit your research? Choose BMC and benefit from:

- fast, convenient online submission

- thorough peer review by experienced researchers in your field

- rapid publication on acceptance

- support for research data, including large and complex data types

- gold Open Access which fosters wider collaboration and increased citations

- maximum visibility for your research: over $100 \mathrm{M}$ website views per year

At BMC, research is always in progress.

Learn more biomedcentral.com/submissions 